\title{
Toints
}

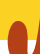

\section{Two cases of chronic knee pain caused by unusual injuries to the popliteus tendon}

\author{
ERIC A. DAVALOS, DAVID BARANK, RAJEEV K. VARMA \\ Department of Radiology, Harbor-UCLA Medical Center, Torrance, USA
}

\begin{abstract}
Injuries to the popliteus tendon are less frequent than injuries to the menisci or ligamentous structures of the knee. When they do occur, injuries to the popliteus tendon tend to be the result of trauma and associated with injuries to other components of the knee. The most commonly seen injuries include tears at the musculotendinous junction and avulsion tears at the lateral femoral condyle insertion site. This report presents two unusual injuries of the popliteus tendon in patients with chronic knee pain: an isolated split tear of the tendon and a subluxed tendon residing within the lateral joint space.
\end{abstract}

Keywords: popliteus tendon, knee, split tear, posterolateral corner, subluxation.

\section{Introduction}

The popliteus tendon is the tendon of the popliteus muscle and is located at the posterior aspect of the knee. It originates from the lateral femoral condyle, where the popliteofibular ligament comprises a portion of its proximal thickness. After passing beneath the lateral collateral ligament, the popliteus tendon runs inferiorly and medially to insert on the posterior medial aspect of the tibia. The popliteus muscle is a component of the posterolateral corner of the knee and functions as a primary restraint to external rotation, a secondary restraint to varus stress, and an internal rotator of the tibia (1). Injuries to the popliteus tendon are usually due to either

\section{Corresponding Author:}

Eric A. Davalos, MD

Department of Radiology, Harbor-UCLA Medical Center 1000 W Carson St Box 27, 90502 Torrance, USA

E-mail: edavalos@dhs.lacounty.gov acute trauma or chronic overuse. In the case of trauma, they tend to be caused by falling on an extended knee, or by forced hyperextension. Isolated injury to the popliteus tendon is very rare and few cases are reported in the literature. Injuries to the popliteus tendon are typically associated with injuries to the posterior cruciate or anterior cruciate ligament and menisci $(2,3)$. In this article we describe two patients with unusual injuries of the popliteus tendon - an atraumatic, isolated split tear, and a medially displaced popliteus tendon residing within the lateral intercondylar space.

\section{Case 1}

A 54-year-old woman presented with a six-month history of right knee pain and swelling. She denied any associated trauma and described the pain as increased when walking and when performing activities of daily living. Physical examination was notable for a normal range of motion, lateral swelling and tenderness to palpation. Plain films of the right extremity demonstrated mild degenerative changes and evidence of retropatellar effusion. The patient was treated with a combination of ibuprofen, acetaminophen and tramadol. Two months later she presented to the Emergency Department with persistent pain. Plain films of the knee were negative and an MRI scan was ordered for a more in-depth assessment.

MRI of the right knee revealed a split tear of the popliteus tendon at its attachment on the lateral femoral condyle (Fig. 1) and a bone contusion at the lateral femoral condyle. There was no injury to the menisci or ligaments.

\section{Case 2}

A 46-year-old woman presented with a two-month history of non-improving left anterior knee pain. The 
pain had developed after she had fallen out of a tow truck onto her knees. She described her left knee pain as a constant, throbbing pain that worsened on knee flexion and when walking or going down stairs. Her physical examination was notable for tenderness to palpation at the superolateral aspect of the patella. She was able to perform single leg squats effectively on the right knee, but with limited ability on the left. At a first assessment six weeks earlier, plain films of the knees had been negative and she had been diagnosed with
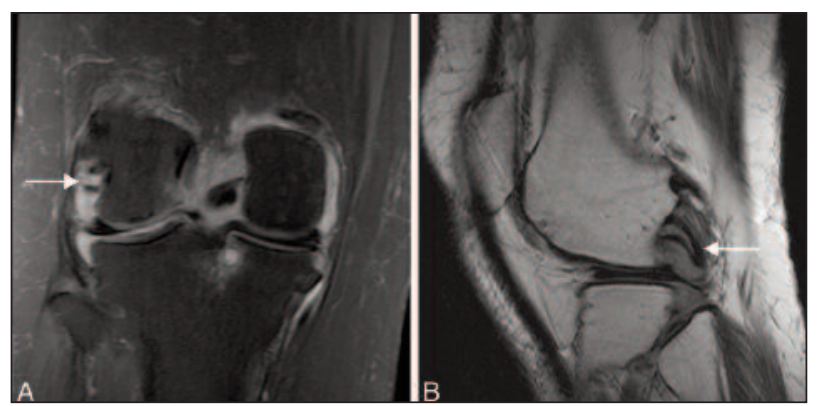

Figure 1. Coronal proton density with fat saturation (a) and sagittal T2-weighted (b) images of a 54-year-old female with a split tear at the proximal portion of the popliteus tendon (white arrows) surrounded by edema.
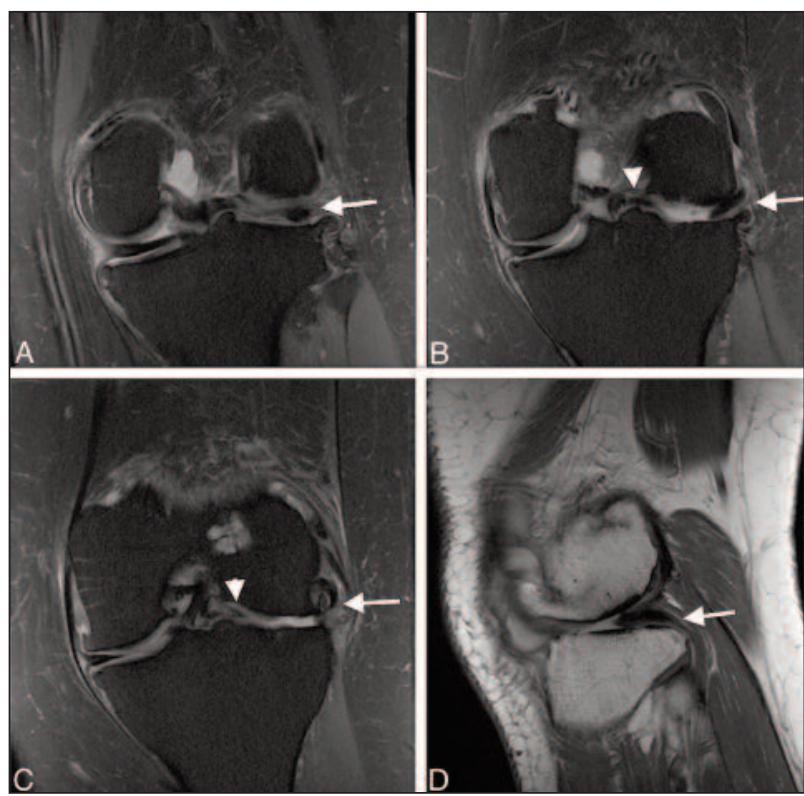

Figure 2. Sequential coronal proton density with fat saturation images, from posterior to anterior (a-c), of a 46-year-old female with a medially displaced popliteus tendon (white arrows) residing within the lateral intercondylar space and a medially displaced lateral meniscus (white arrowheads). Sagittal proton density (d) weighted image also shows the popliteus tendon in the lateral intercondylar space (white arrow). patellofemoral pain syndrome. An MRI scan was ordered for a more in-depth evaluation. MRI of the left knee revealed a medial meniscus tear with a multilobulated, septated perimeniscal cyst arising from the anterior horn of the medial meniscus. There was also a complex tear of the lateral meniscus with extrusion of meniscal fragments into the intercondylar notch and lateral gutter. The popliteus tendon appeared to originate and attach normally, however it followed an abnormal path between the lateral femoral condyle and lateral tibial plateau (Fig. 2).

\section{Discussion}

The popliteus tendon is a component of the complex posterolateral corner of the knee. The other structures of this region are: the fibular collateral ligament, the popliteofibular ligament, the fabellofibular ligament, the arcuate ligament, the middle third lateral capsular ligament and the biceps femoris tendon (1). The primary function of the popliteus tendon is to internally rotate the knee, rotating the tibia medially on the femur. The popliteus tendon and popliteofibular ligament have also been reported to be the most important structures for preventing external tibial rotation (3). Injury to the muscle-tendon unit of the popliteus should be suspected in patients with an acutely swollen knee and lateral knee pain (4).

Typical injuries to the popliteus tendon include femoral avulsion or musculotendinous ruptures. The vast majority of popliteus tendon injuries are associated with other lateral/posterolateral ligamentous or meniscal injuries, while isolated injuries of the popliteus tendon account for fewer than $10 \%(2,4-7)$.

A review of the literature reveals several reports documenting isolated avulsion or complete rupture injuries of the popliteus tendon $(4,8-17)$ and only two reports of an isolated partial popliteus tendon tear $(6,18)$. Furthermore, most cases of isolated avulsion of the popliteus tendon without an associated osteochondral fragment tend to lack definitive image findings and are subsequently diagnosed on arthroscopy.

The presence of a bifurcated popliteus tendon, which is a normal variant finding, was another possibility considered in our differential diagnosis of case 1 . In 2009, Leal-Blanquet et al. reviewed 1,569 arthrosco- 
pies and found six cases with a bifurcated popliteus tendon. All six cases were asymptomatic patients who presented no supportive pre-arthroscopy MRI findings (19). Given that our patient was symptomatic and had tendinous separation significant enough to be detected on MRI, we believe her findings represent a true tear. She was referred to the Orthopaedic Department where it was recommended that she receive a steroid injection and conservative management with physical therapy.

The patient in case 2 had an abnormally located popliteus tendon situated between the femur and tibia, a finding that has, to our knowledge, not previously been described. Further evaluation of the posterolateral corner also revealed a medially displaced lateral meniscal body extending into the intracondylar notch. We speculate that this displaced meniscus, by creating tension that had the effect of pulling the tendon into the meniscal space, could have been a factor contributing to the abnormal path of the popliteus tendon. We further speculate that this anomaly could also have been due to a non-visualized rupture of the popliteofibular ligament which may have been disrupting the lateral tension naturally created by the tendon. The extent to which the abnormally positioned popliteus tendon was contributing to this patient's pain is difficult to discern in view of her associated meniscal tears, but it seems likely that this abnormality was a significant component of her pain given that, on MRI interpretation at least, the patient appeared to be walking on her popliteus tendon. The patient was referred to the Orthopaedic Department, however she was never seen and was lost to follow-up.

\section{References}

1. Pacholke DA, Helms CA. MRI of the posterolateral corner injury: a concise review. J Magn Reson Im. 2007;26:250-255.
2. Recondo JA, Salvador E, Villanúa JA, et al. Lateral stabilizing structures of theknee: Functional anatomy and injuries assessed with MR imaging. Radiographics. 2000;20(special issue):S91-S102.

3. Haims AH, Medvecky MJ, Pavlovich R Jr, et al. MR imaging of the anatomy of and injuries to the lateral and posterolateral aspects of the knee. AJR Am J Roentgenol. 2003;180:647-653.

4. Guha AR, Gorgees KA, Walker DI. Popliteus tendon rupture: a case report and review of the literature. Br J Sports Med. 2003;37:358-360.

5. Blake SM, Treble NJ. Popliteus tendon tenosynovitis. Br J Sports Med. 2005;39:e42.

6. Mariani PP, Margheritini F. Partial isolated rupture of the popliteus tendon in a professional soccer player: a case report. Sports Med Arthrosc Rehabil Ther Technol. 2009;1:18.

7. Brown TR, Quinn SF, Wensel JP, et al. Diagnosis of popliteus injuries with MR imaging. Skeletal Radiol. 1995;24:511-514.

8. Burstein DB, Fischer DA. Case report: isolated rupture of the popliteus tendon in a professional athlete. Arthroscopy. 1990;6:238-241.

9. McConkey JP. Avulsion of the popliteus tendon. J Pediatr Orthop. 1991;11:230-233.

10. Nakhostine M, Perko M, Cross M. Isolated avulsion of the popliteus tendon. J Bone Joint Surg Br. 1995;77:242-244.

11. Mirkopulos N, Myer TJ. Isolated avulsion of the popliteus tendon. A case report. Am J Sports Med. 1991;19:417-419.

12. Garth WP Jr, Pomphrey MM Jr, Merrill KD. Isolated avulsion of the popliteus tendon: operative repair. A report of two cases. J Bone Joint Surg Am. 1992;74:130-132.

13. Naver L, Aalberg JR. Avulsion of the popliteus tendon. A rare cause of chondral fracture and hemarthrosis. Am J Sports Med. 1985;13:423-424.

14. Gruel JB. Isolated avulsion of the popliteus tendon. Arthroscopy. 1990;6:94-95.

15. Westrich GH, Hannafin JA, Potter HG. Isolated rupture and repair of the popliteus tendon. Arthroscopy. 1995;11:628-632.

16. Conroy J, King D, Gibbon A. Isolated rupture of the popliteus tendon in a professional soccer player. Knee. 2004;11:67-69.

17. Murray JR, Grundy JR, Collins IE, et al. Spontaneous rupture of the popliteus tendon in a 74-year-old woman and review of the literature. Arthroscopy. 2004;20:860-864.

18. Rose DJ, Parisien JS. Popliteus tendon rupture: case report and review of the literature. Clin Orthop Relat Res. 1988; (226):113-117.

19. Leal-Blanquet J, Ginés-Cespedosa A, Monllau JC. Bifurcated popliteus tendon: a descriptive arthroscopic study. Int Orthop. 2009;33:1633-1635. 